\title{
Simulation of Fuzzy Traffic Light Controller Using Fuzzytech5.72
}

\author{
Rajeev ${ }^{1}$, Pawan Kumar Pandey ${ }^{2}$, Parveen Kumar ${ }^{3}$ \\ ${ }^{1,3}$ (Research Scholar EEE Department, BRCMCET, Bahal, Bhiwani, Haryana-127028, India) \\ ${ }^{2}$ (Faculty Member EEE Department, BRCMCET, Bahal, Bhiwani, Haryana-127028, India)
}

\begin{abstract}
The concentration of vehicles on road is increasing day by day throughout the world particularly in large urban areas. Due to this traffic congestion problem is also increasing. To accommodate this increasing demand an optimized method for traffic control is required. As the transportation system will grow the need for an intelligent controller will have to be employed to face controlling the traffic congestion problems. Fuzzy controllers have been universally used for many industrial applications but for traffic controls it hasn't been widely applied. This paper proposes the use of fuzzy based traffic light controller using FUZZY TECH software at a complex traffic junction. The real time parameters such as traffic density and queue length are obtained by using image processing techniques. So the on and off timings for the green, red and orange lights are adjusted as per the actual road conditions. In this paper a 4 way intersection fuzzy traffic controller has been described suitable for mixed traffic. The rule base has been formulated by human experts, fuzzy inference engine and the defuzzifier.
\end{abstract}

Keywords: Traffic Control, Fuzzy Logic, membership function, Intelligent Transportation Systems (ITS), Defuzzification

\section{Introduction}

Traffic congestion problem is a very big problem in many developed as well as developing countries. Many critical problems are being caused due to this traffic congestion. The traveling between two different places is becoming more and more difficult. Due to these problems people lose time, miss opportunities and get frustrated. This problem also has a worse impact on companies. The productivity goes on declining, delivery gets delayed and as a result the cost of product increases. As this problem will go on increasing with time the new facilities and infrastructure has to be build. But the disadvantage of making new roads is that the surroundings become more congested. The main goal of this paper is to minimize traveling time, improving safety and increasing the capability of the infrastructure. The traffic control is highly dependent on elements like time, day, season weather and unpredictable situations such as road accidents, any event or construction work going on. A traffic control system which solves all the above mentioned problems is called and intelligent control system. But in this system the main problem is that that all the traffic control is generally dependent upon the operator's judgment. The problems which we have to face in case of human traffic control are:

1. The suitable decisions can only be made by the skilled person.

2. The work load on the human operator is very high so the decisions taken by him/her cannot be the appropriate one.

3. To solve this problem, it becomes necessary to formulate the operator's judgment and then to develop an automatic decision making system.

Today's Technique

1. Microcontrollers control all the functioning.

2. The control is not flexible based on the condition of traffic and crossing.

3. There are no real time adjustments of the on/off timings as per the traffic conditions and crossings.

This paper presents possible solutions to all the above mentioned problems using the fuzzy logic.

\section{The Proposed Technique}

4. Real time adjustments are present in this technique to reduce the traveling time.

5. Flexible control is possible based on the condition of traffic and crossing.

6. Simulations are used to examine and analyze the effectiveness of the proposed FLSC. 


\section{Design Criteria}

7. A 4 way isolated junction with traffic coming from north, west, south and east directions has been considered.

8. At the instant when the traffic coming from north and south is moving, traffic from the west and east stops and vice versa.

9. There are no left and right turns are included.

10. The fuzzy logic controller will observe the density of the north and south traffic as one side and west and east traffic as another.

11. The east west lane is assumed as the main approach.

\section{Design for Fuzzy Logic Traffic Light Controller}

The input variables are:

a) Quantity of the traffic on the arrival side (Arrival);

b) Quantity of the traffic on the queuing side (Queue).

If the north and south sides is green then it would be considered as the arrival sides while the north and the south side would be considered as queuing side, and vice versa. The following points are required to be remembered:

The four traffic lights work in four sequences, every light having a variable sequence from 10 to 130 seconds depending upon the congestion.

All the four traffic lights will be controlled by the same mechanism.

\section{Simulation Process}

On double clicking the Fuzzytech5.72 icon the Project Editor window appears. After this the process followed is as described further:

Call the FDW by either selecting "File/Fuzzy Design Wizard..." in the main menu or by clicking the respective button in the main toolbar. Follow the instructions given on the following pages.

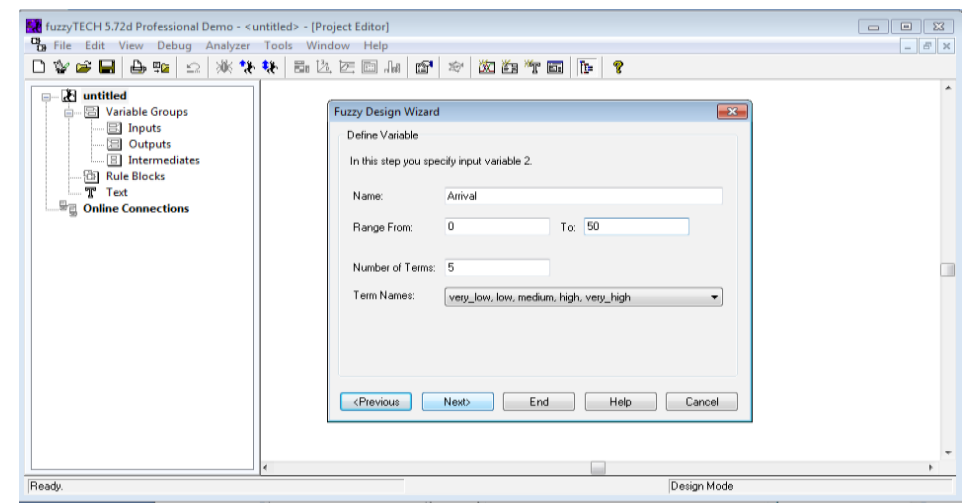

Figure 1Welcome Dialog of the Fuzzy Design Wizard

1. Enable the check box, "Create New System" (see Figure 1). Press the [Enter] key or click the [Next>] button to open the next FDW dialog (Figure 2).

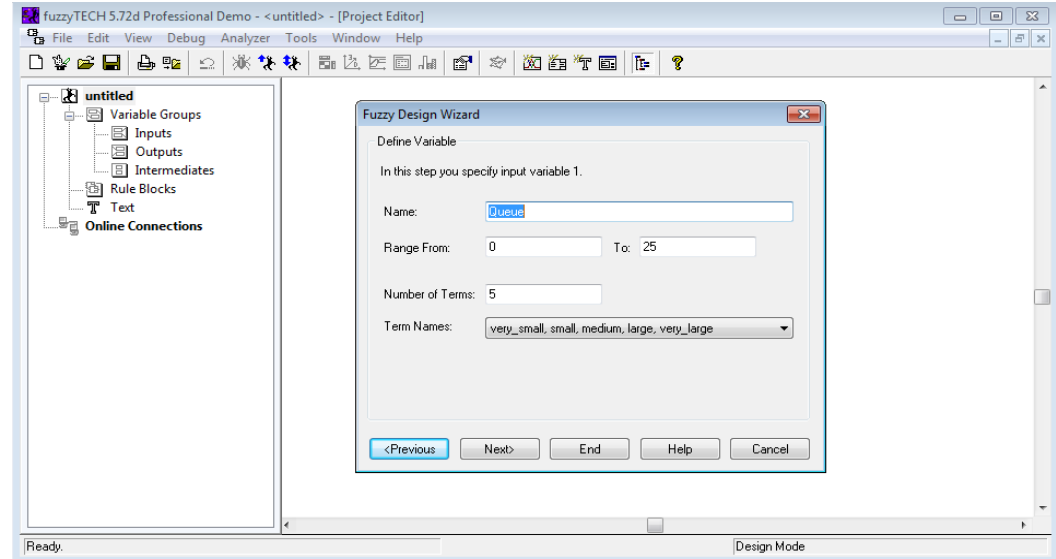

Figure 2 In the Second FDW Window, You Specify the Number of Input, Output and Intermediate Variables and Their Term Numbers. 
2. Accept the suggested default settings and click the [Next $>$ ] button to open the next FDW dialog (Figure 3).



Figure 3 : The FDW Opens This Dialog for Each Variable to be created.

3. Accept the settings proposed by the FDW and step to the next FDW dialog. The dialog for defining the next variable opens. This is continued as long as there are input, intermediate and output variables of the system to be defined. After all variable have been defined, the next FDW dialog Define Defuzzification opens. This dialog helps to define the defuzzification method for the output variable. Accept the proposed default by clicking the [Next] button or pressing the [Return] key.

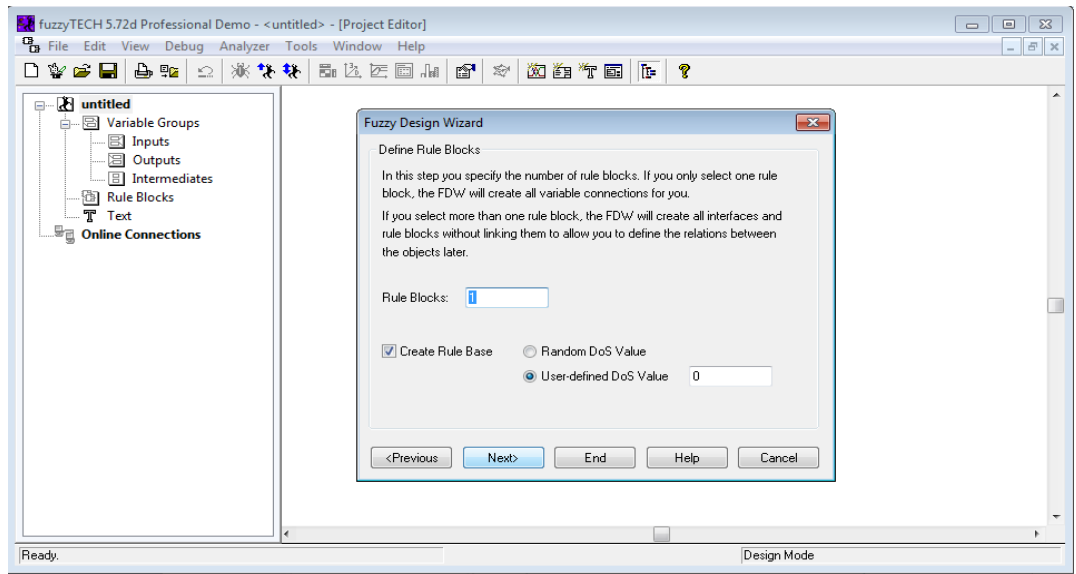

Figure 4 The Last FDW Window Specifies Whether and How the FDW Generates Rule Blocks.

4. Accept the proposed default values of the FDW and either click the [Next] button or press the [Return] key. Since this is the final wizard of the FDW, this is equivalent to pressing the [End] button. After pressing [Next], the Confirmation dialog (Figure 5) appears

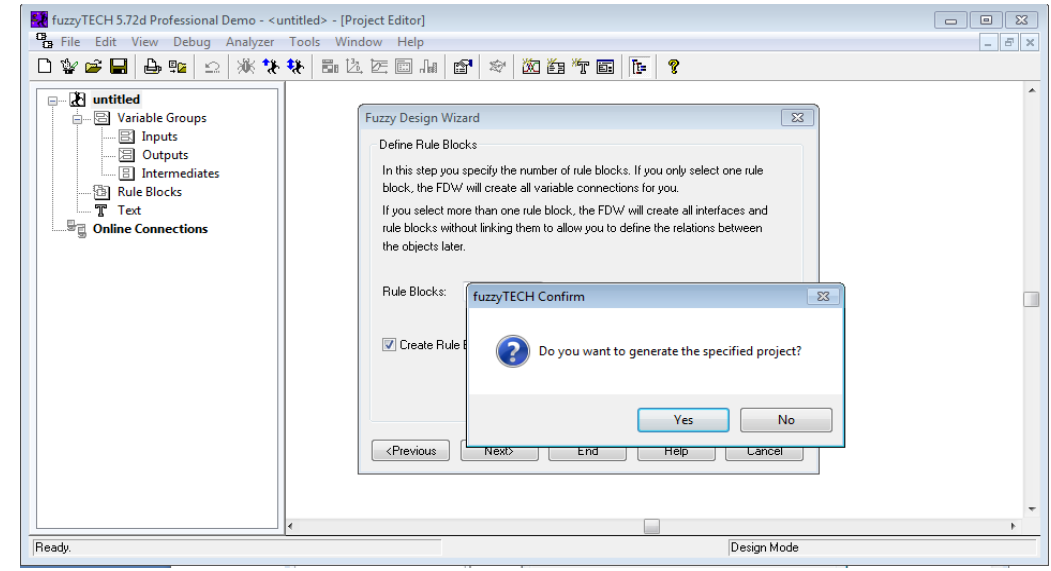

Figure 5 Before the Fuzzy Design Wizard Generates the System, This Confirmation Dialog Is Displayed. 
5. When the input variable blocks in the fuzzy structure are double clicked (figure 6) the input variable membership wizard appears as shown in figure 7 and 8 .

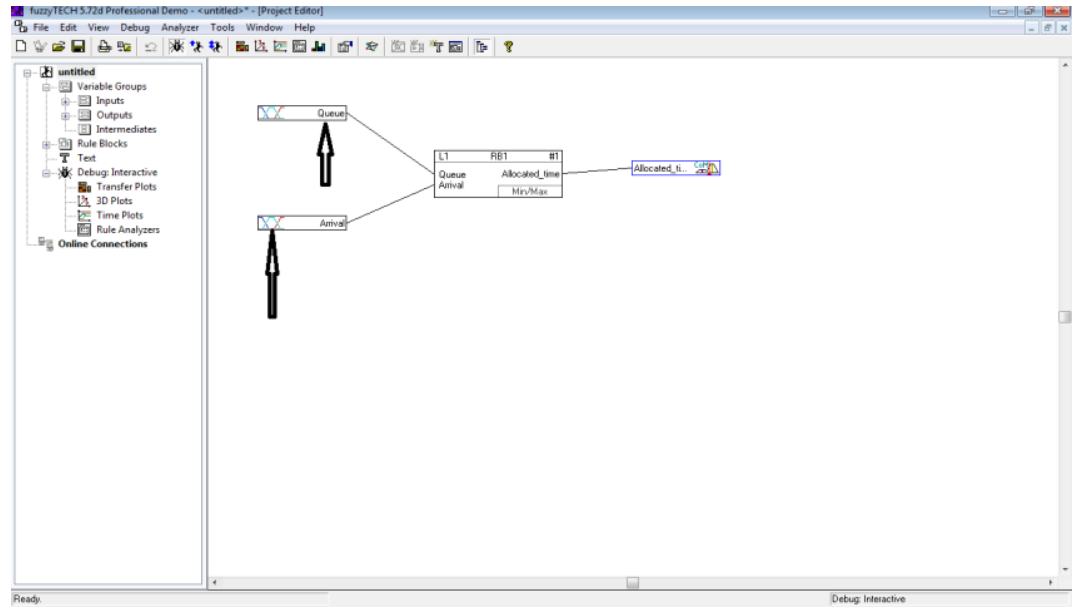

Figure 6 Structure of the System Generated By the FDW.

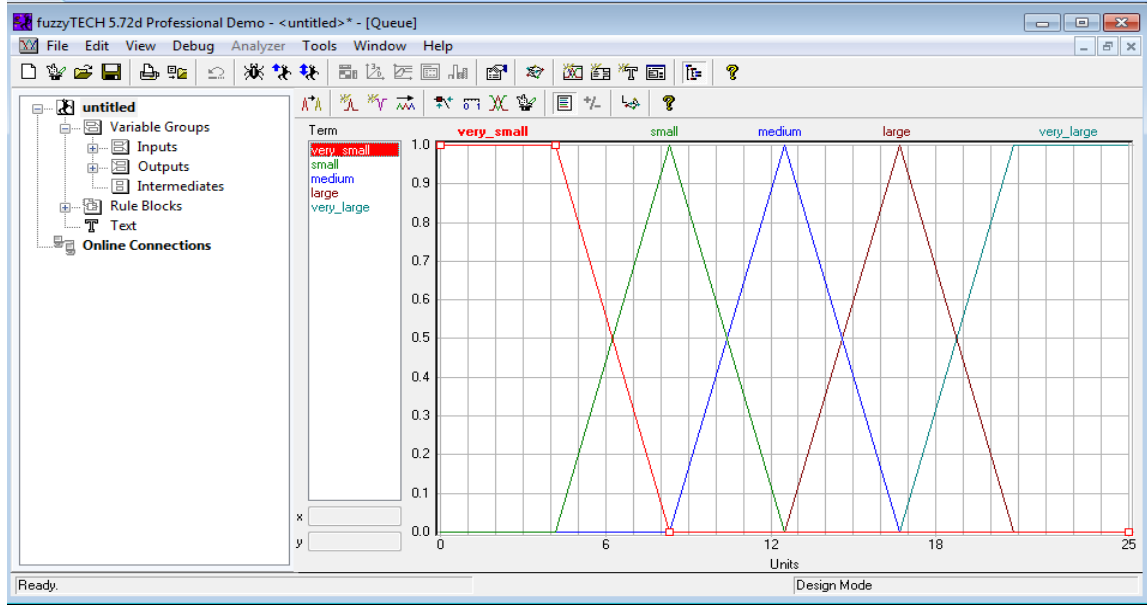

Figure 7: Defining a Standard MBF for the input variable "Queue”.

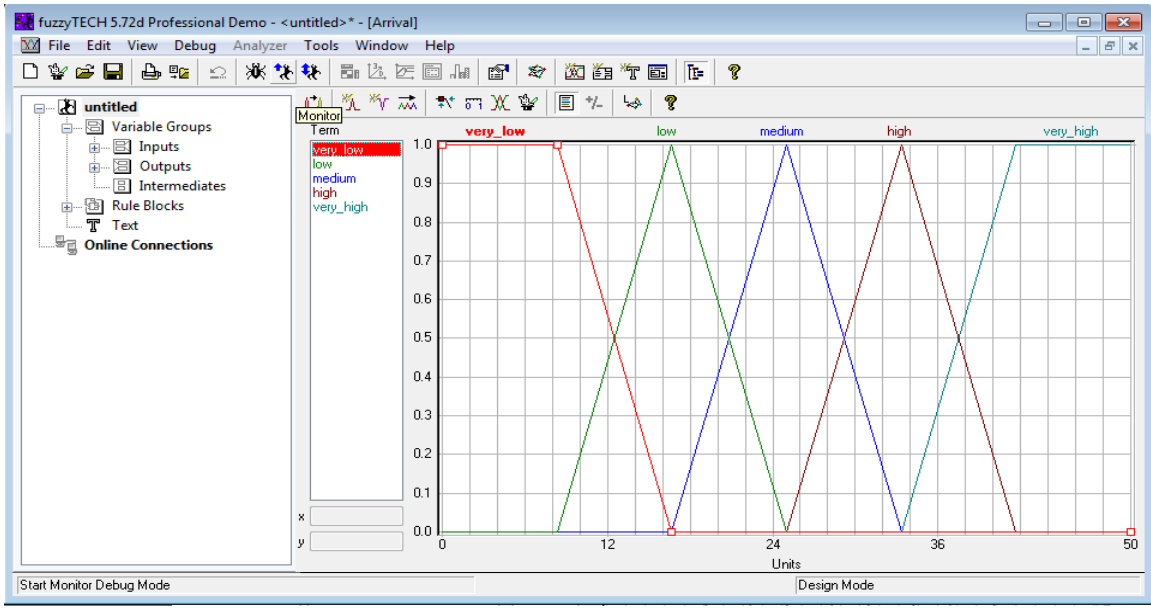

Figure 7 Defining a Standard MBF for the input variable "Arrival". 
6. Enter your production rules in either the Spreadsheet Rule Editor or the Matrix Editor (Create at least one rule). At first, open the Spreadsheet Rule Editor by double-clicking a rule block in the Project Editor or a rule block's name in the Tree view.

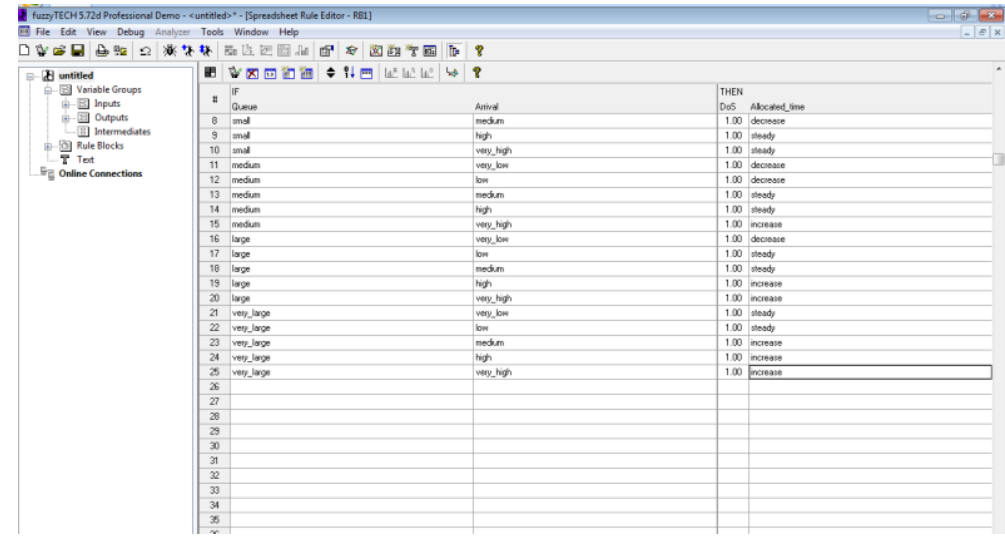

Figure 8 Rule Block Properties: Operators Dialog

7. Similarly when the output variable block in the fuzzy system structure wizard is double clicked the output variable wizard appears as shown in figure 10.

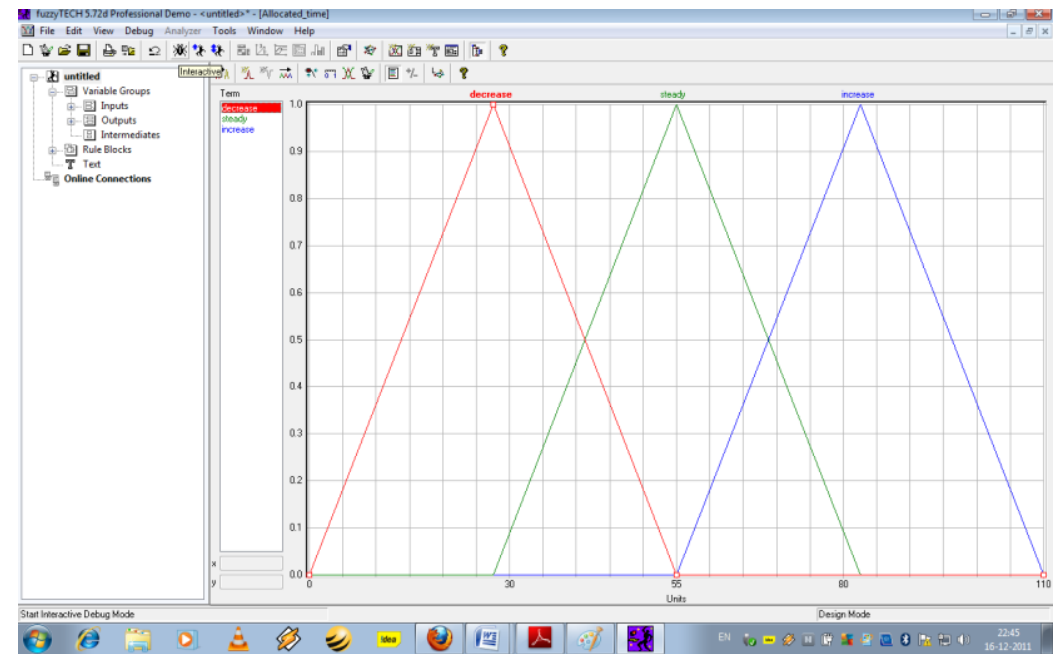

Figure 9 Defining a Standard MBF for the output variable "Allocated_time".

8. Switch into Interactive debug mode by selecting "Debug/Interactive" or click the symbol [Interactive] in fuzzyTECH's main toolbar. In this mode we can specify the value of input variables according to our need.

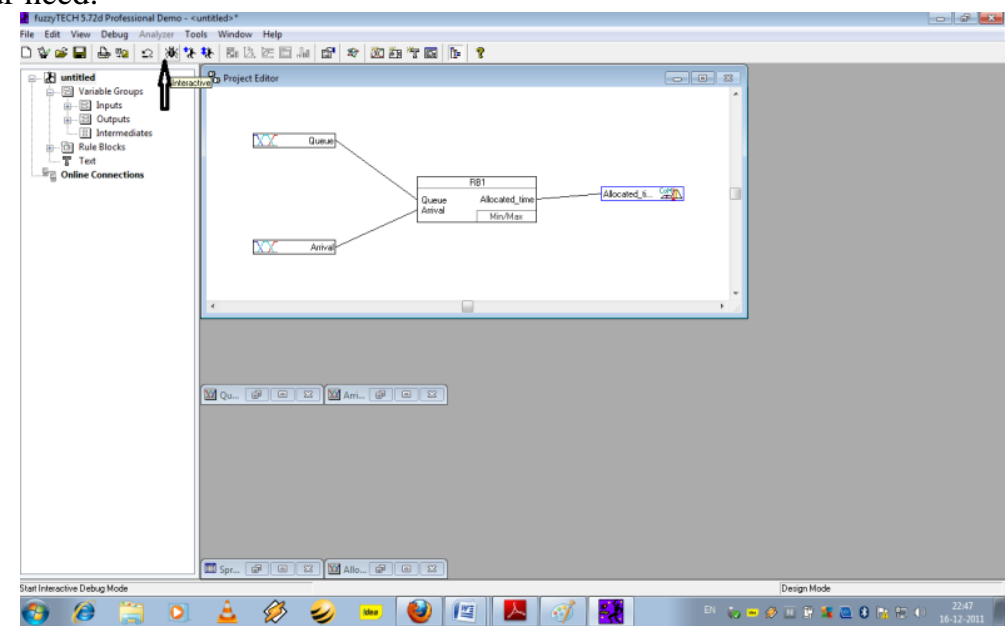

Figure 10 Defining how to put the system structure in debug mode. 
9. The input variables can be changed according to the need in the watch window wizard and the corresponding variations in the output can also be observed here.

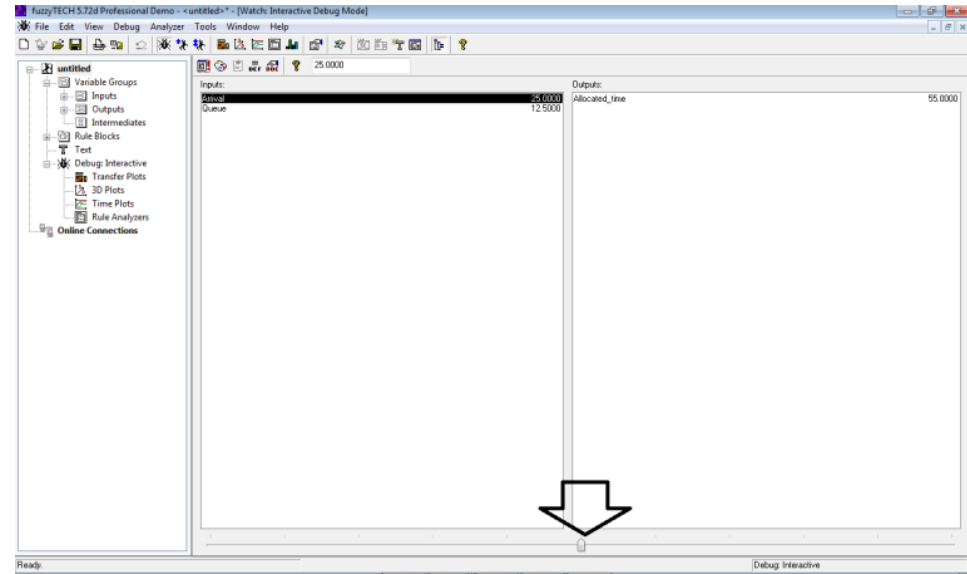

Figure 11 The Watch Window Shows All I/O Variables and Their Values.

10. In debug mode of fuzzy system structure (figure 13) when the input variable blocks are double clicked again the input variable membership wizard appears as in the earlier cases but in this mode the value of input variables can now be changed when the slider is moved from left to right or vice versa as indicated in figure 14 and 15 .

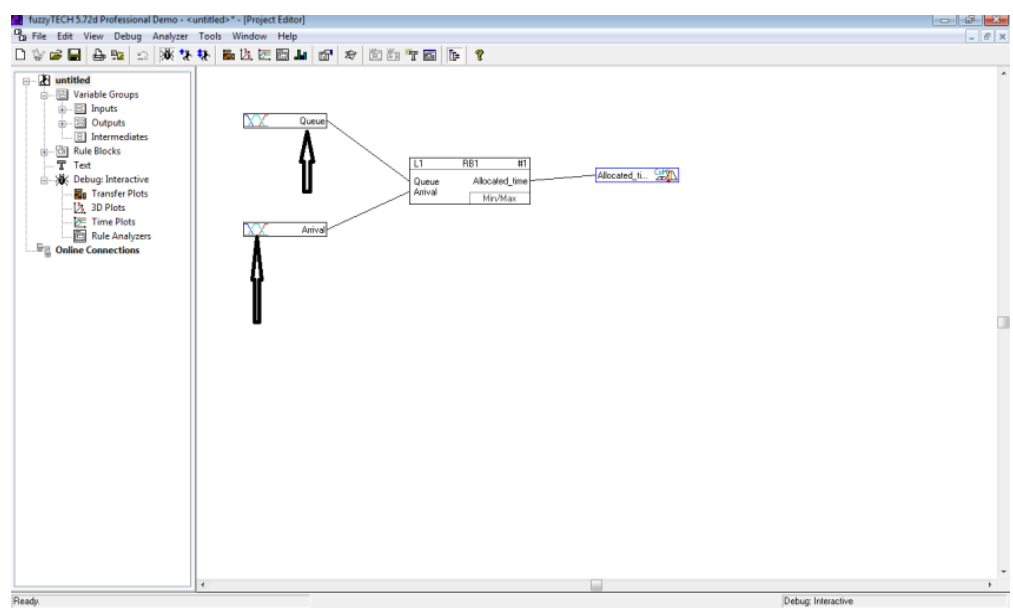

Figure 12 The Fuzzy System Structure in Debug Mode.

11. Specify input value "Arrival" for the actual system. Review the system behaviors by visualizing the entire fuzzy inference in the fuzzyTECH editors.

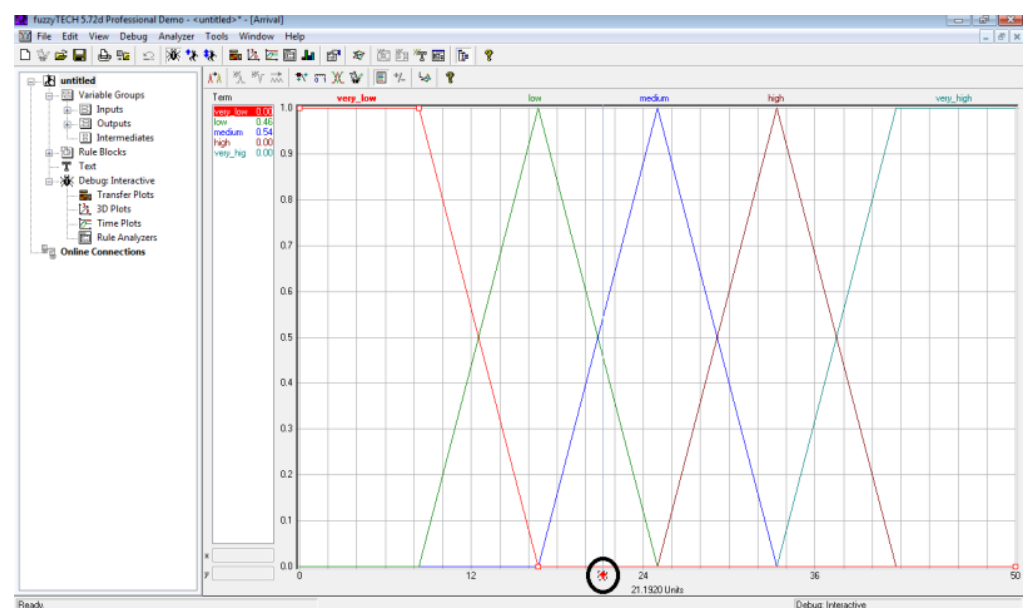

Figure 13 Defining a Standard MBF for the input variable "Arrival" in debug mode. 
12. Specify input value "Queue" for the actual system. Review the system behaviors by visualizing the entire fuzzy inference in the fuzzyTECH editors.

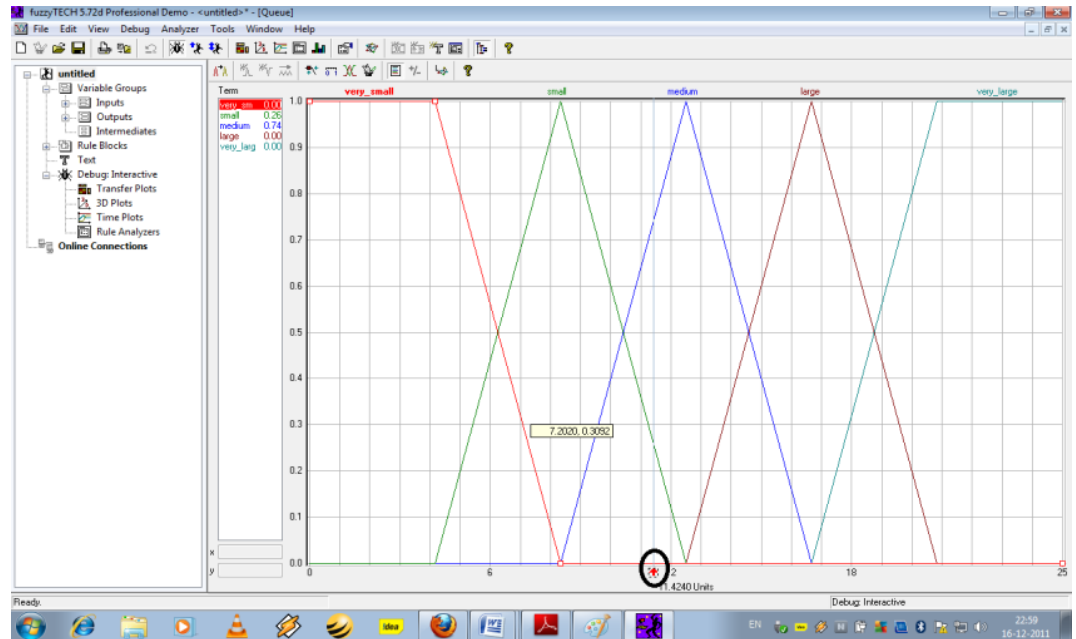

Figure 14Defining a Standard MBF for the input variable "Queue" in debug mode.

13. This window appears when the output block in the fuzzy system structure during debug mode is double clicked. The change in output variable (Allocated_time) can be observed here.

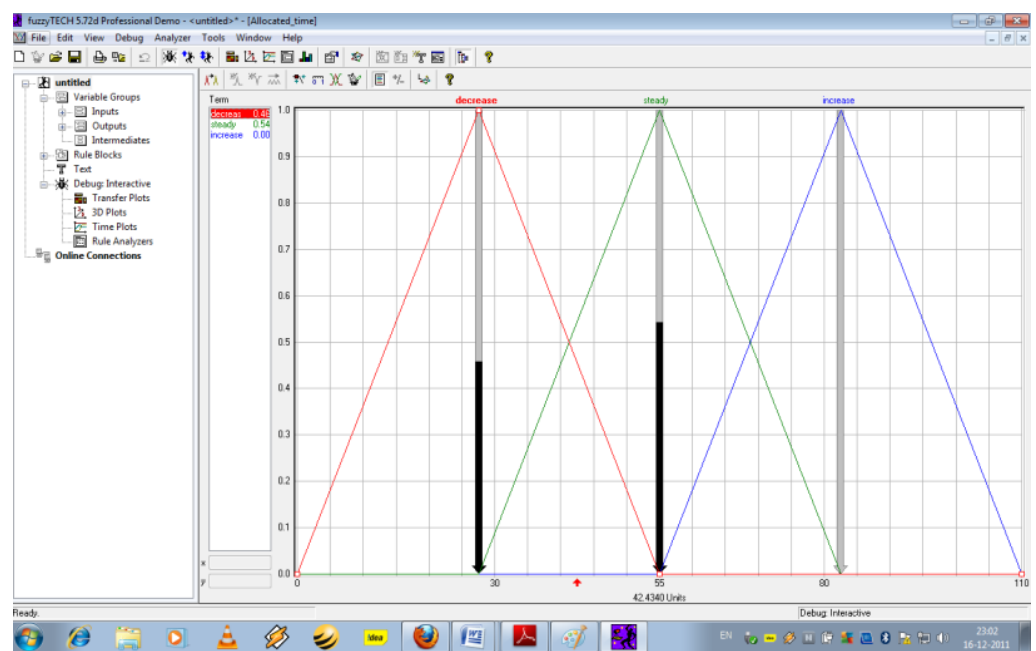

Figure 15Defining a Standard MBF for the output variable "Allocated_time" with corresponding change in output.

14. In the analyzer/transfer plot wizard the transfer plot of simulation process can be seen (figure 17).

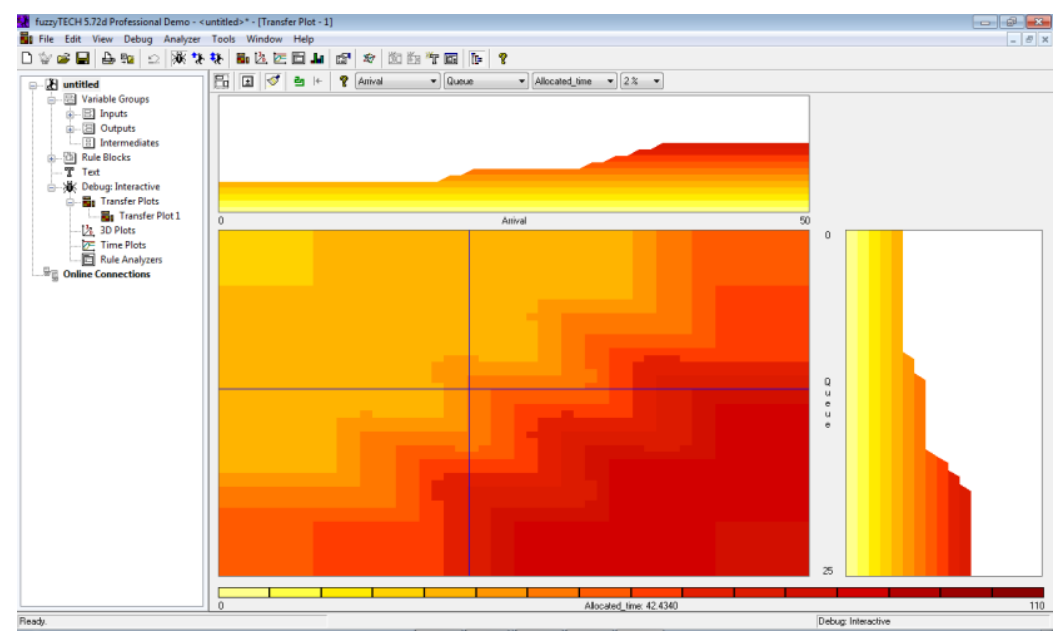

Figure 16Transfer Plot Analyzer Window 
15. In the analyzer/rule analyzer plot wizard the rule analyzer can be seen (figure 18).

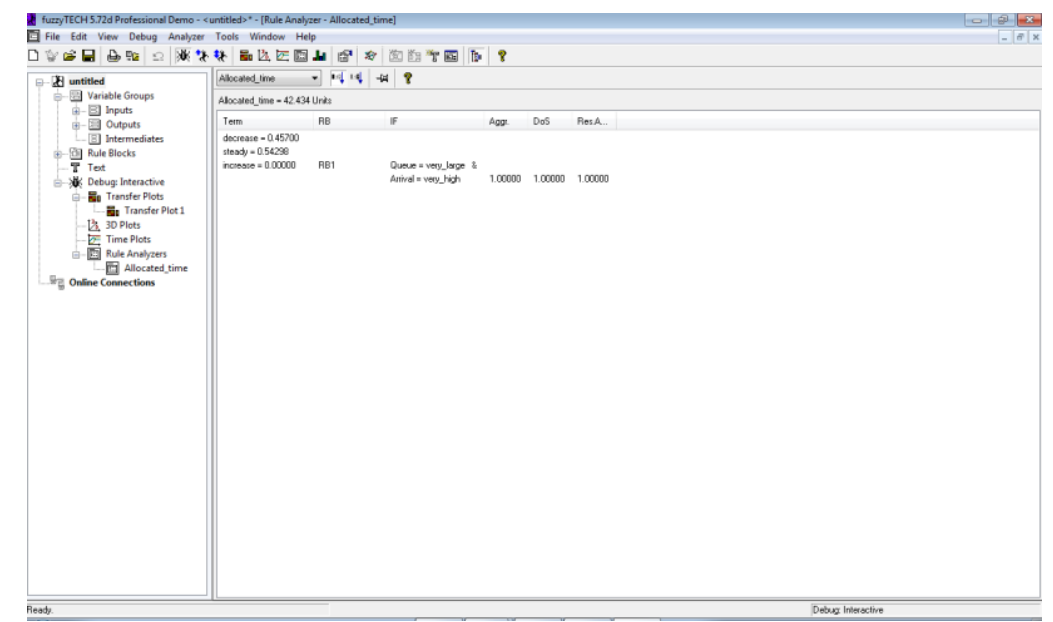

Figure 17Rule Analyzer for Output Variable "Allocate_time"

16. In the analyzer/3D plot wizard the $3 \mathrm{D}$ plot of simulation process can be seen (figure 19).

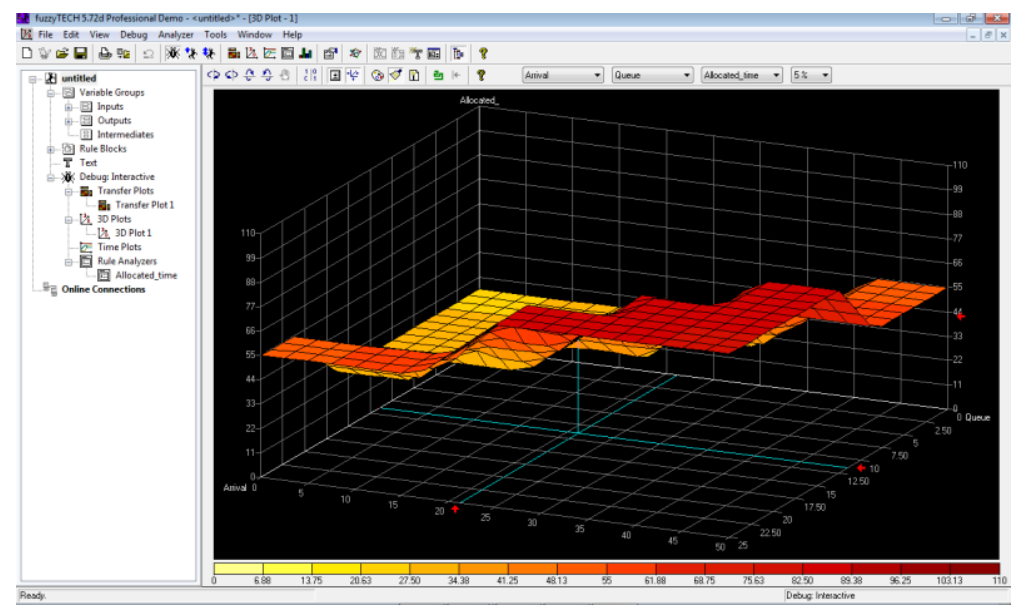

Figure 183D Plot

\section{Conclusion and Future Works}

In this paper the system presented is very flexible. The status of Queue can easily be observed from images taken from cameras. The fuzzy logy can effectively deal with the uncertainty; it can be more advantageous for the traffic light systems. A suitable on/off time of lights is provided by this system which makes the infrastructure capacity more effective and the vehicles do not require waiting for too long. Less waiting time and less congestion also reduce the fuel consumption and sound pollution and also the time and energy wastage. More input variables like weather conditions and other environment conditions can be added to have more précised traffic control.

\section{References}

17. Akçelik, R., Besley, M., Roper, R., "Fundamental Relationships for Traffic Flows at Signalized Intersections, ARRB Transport Research Ltd.," Research Report No. 340, pp. 268, ISBN 0869107968, 1999

18. Hegyi, B. De Schutter, R. Babuska, S. Hoogendoorn, H. VanZuylen, H. Shuurman, "A Fuzzy Decision Support System for Traffic Control Centers[J]", Faculty of Information Technology and Systems, Aug. 2001, pp.358-363.

19. Beauchamp-Baez,G., Rodriguez-Morales, E., Muniz Marrero, E.,L., "A Fuzzy Logic Based Phase Controller for Traffic Control", Proc. IEEE International Conference on Fuzzy Systems 1997, pp.1533-1539.

20. Dr. Devinder Kaur, Elisa Konga, Esa Konga, "Fuzzy Traffic Light Controller, Circuits and Systems" 1994, Proceedings of the 37th Midwest Symposium pp.1507-1510 Vol.2, August,1994.

21. Xu Li-qun, Jiang Fu, "Fuzzy Reasoning Algorithm for Control of Traffic in Congestion Route[J]", Information and Control, Apr.2003, Vol.32, No.2, pp. 132-141. 